VA Medical Center/University of Michigan Ann Arbor, Michigan 48105

\section{Elsewhere Reviews}

\section{LOCALIZING GLUCOSE TRANSPORT PROTEINS: ACTIVE INVESTIGATION OF PASSIVE CARRIERS}

Thorens B, Cheng Z-Q, Brown D, Lodish $H F$. Liver glucose transporter: a basolateral protein in hepatocytes and intestine and kidney cells. Am J Physiol 1990;259: C279-C285.

\section{ABSTRACT}

The "liver" isoform of the facilitated diffusion glucose transporter is expressed predominantly in liver, intestine, kidney, and pancreatic islet $\beta$-cells. The apparent molecular mass of the transporter in liver, kidney, and intestine is different, as detected by Western blot analysis of membrane proteins using antipeptide antibodies. However, as assessed by Northern blot analysis and molecular cloning, the same mRNA is expressed in these tissues, indicating that there are tissue-specific posttranslational modifications of the same transporter polypeptide. As determined by immunofluorescence analysis on frozen tissue sections, the liver glucose transporter is present on the sinusoidal membrane of hepatocytes, on the basolateral membrane of fully differentiated absorptive intestine epithelial cells, and on the basolateral membrane of proximal tubule cells of the kidney nephron. This localization is consistent with the involvement of the liver glucose transporter in several key steps of glucose metabolism: glucose uptake and release by the liver and absorption or reabsorption by epithelial cells of the intestine and kidney, respectively.

Thorens B, Lodish HF, Brown D. Differential localization of two glucose transporter iso-forms in rat kidney. Am J Physiol 1990;259:C268-C294.

\section{ABSTRACT}

The localization of two glucose transporter isoforms was mapped in the rat kidney: the high-Michaelis constant $\left(K_{m} ; 15-20 \mathrm{mM}\right)$ low-affinity "liver" transporter and the low- $K_{m}(1-2 m M)$ high-affinity erythroid/brain transporter. Both are basolateral membrane proteins, but the liver transporter was present exclusively in the $S_{x}$ part of the proximal tubule, whereas the erythroid/brain transporter was expressed at variable levels in different nephron segments. Staining intensity was low in the straight proximal tubule $\left(S_{3}\right)$, intermediate in the medullary thin and thick ascending limbs, and highest in connecting segments and collecting ducts. In the collecting duct, the erythroid/brain glucose transporter was expressed at the highest level in intercalated cells; less was present in principal cells. In the papilla, only intercalated cells expressed this transporter isoform. These results suggest specific involvement of each transporter isoform in transepithelial glucose reabsorption by different segments of the proximal tubule. They also indicate that while the liver glucose transporter is present in gluconeogenic cells, there is a good correlation between the level of expression of the erythroid/brain glucose transporter and the glycolytic activity of the different nephron segments.

\section{COMMENTS}

Glucose, an essential, water-soluble nutrient, requires specific transport proteins to traverse the plasma membrane's hydrophobic core. Thus far, two distinct classes of membrane-embedded glucose transport proteins have been identified. One type actively transports glucose against its concentration gradient, energized by the simultaneous downhill transport of sodium. The more frequently encountered form of glucose-carrier protein passively catalyzes glucose transport down its concentration gradient, a process termed facilitated diffusion. These passive glucose transporters or carriers are found in a variety of cells and are involved with import or export of glucose across the plasma membrane. In glucose-transporting epithelia such as intestine and kidney, the passive glucose transport proteins have been thought to be present in the basolateral membrane, facilitating exit of glucose pumped into the cell by brush-border membrane-active glucose transporters. Passive glucose carriers also enable glucose entry into cells for glycolysis or permit exit of glycogenolytic or gluconeogenic products. Interestingly, the active and passive glucose transport proteins, with the exception of sharing primary substrate and being located in the plasma membrane, are distinct in terms of inhibitors, structural specificity of substrates and primary structure (1-3).

Cells whose main function is to metabolize glucose taken up from extracellular fluid such as erythrocytes and brain have a high-affinity (uptake $\mathrm{K}_{\mathrm{m}}=1$ to $2 \mathrm{mmol} / \mathrm{L}$ ) transporter that would be nearly saturated at a typical plasma glucose concentration of $5 \mathrm{mmol} / \mathrm{L}$. Adipocyte glucose uptake also occurs by way of a related system, which differs from the erythrocyte system in that $\mathrm{V}_{\max }$ is regulated by insulin (4). In contrast, liver sinusoidal membranes contain a low-affinity (uptake $\mathrm{K}_{\mathrm{m}}>20 \mathrm{mmol} / \mathrm{L}$ ) glucose transporter (5). Glucose can move in either direction across the hepatocyte sinusoidal membrane, with efflux occurring in the fed state, when intracellular concentrations can exceed those in plasma (6). Intestinal basolateral membranes and a few other 
tissues share a similar low-affinity glucose carrier. In cells with low-affinity glucose transport, glucose movement is primarily efflux because the glucose concentration in these cells surpasses that in plasma.

Corresponding to the three physiological types of glucose transport just described, three major isoforms of the glucose transporter have been cloned. One type is the "erythroid/brain," also termed "brain/HepG2" because clones sequenced from the human HCC cell line HepG2, red cells and brain are nearly homologous (7). The second isoform is the "liver" transporter, obtained from hepatic complementary DNA (cDNA) libraries. It has also been found in pancreatic islet $\beta$ cells, intestine and kidney (8). The third isoform is the "muscle/adipocyte" isoform found in heart, skeletal muscle and fat cells (4). The three isoforms share considerable homology in the putative membrane spanning segments but less so in other regions, resulting in roughly $60 \%$ overall homology between isoforms.

In the articles under consideration, passive glucose transport proteins were immunolocalized in liver, small intestine and kidney with antibodies prepared against synthetic peptides unique to the liver and erythroid/brain isoforms. They confirm the physiological prediction that passive glucose carriers should only exist in blood-facing or basolateral surfaces of epithelia.

In the paper addressing the glucose carrier of liver, intestine and kidney, immunoblots of hepatic, renal and intestinal membranes revealed that protein recognized by glucose antibody differed in molecular mass according to the source tissue. Restriction maps demonstrated that the passive glucose carrier in liver, kidney and intestine, despite differences in protein size, had nearly identical nucleotide sequences and thus belonged to the same "liver" isoform. Immunostaining with "liver" glucose carrier antibodies revealed staining of the sinusoidal membranes, with absence of staining of the canalicular-associated membranes (identified with a second antibody). In the small intestine, glucose carriers were immunolocalized to the basolateral membrane of differentiated surface cells. This finding confirms the notion, inferred from whole-tissue data, that the surface cells, and not the crypt cells, mediate intestinal glucose transport and that passive glucose carriers convey intracellular glucose into the blood. In the kidney, the liver isoform was localized to the basolateral membrane of the proximal convoluted tubule, again, as would be predicted for a protein involved with transepithelial glucose movement.

The second paper examines the distribution of the erythroid/brain glucose transporter isoform in kidney. This isoform was located in the basolateral membranes of cells in a variety of segments of nephron. In the intercalated and principal cells, differentiated by immunostaining with $\mathrm{H}^{+}$-ATPase antibody, staining was most intense in the intercalated cells of the collecting duct.

In discussing their findings, the authors conclude that the liver-type transporter is found in cells that export glucose into blood, whereas the erythroid/brain isoform is found in cells taking up glucose from plasma. Collecting duct intercalated cells, which have high energy requirements because of the presence of plasma membrane proton pumps, thus have the highest density of glucose transporters.

Taken together, the papers represent an important contribution to the understanding of glucose transport across epithelial basolateral membranes. With technology able to unequivocally localize glucose transporter isoforms in tissue, it was possible to discriminate between transporter subtypes in tissues such as kidney, where more than one form exists. Immunolocalization thus has clear advantages in this regard over alternative technologies such as whole tissue perfusions or transport measurements with membrane vesicles, which are generally confounded by the presence of more than one type of carrier. The methodology can also be used to study regulation, such as in recent publications in which cDNA probes and specific antibodies were used to examine glucose carrier regulation in response to regeneration and diabetes $(9,10)$.

A few comments might also be added that in no way diminish the superb contributions these papers represent. The authors propose in the second paper that the presence of the high-affinity liver-type transporter in the $\mathrm{S}_{3}$ segment of proximal tubule implied that these cells might have high-affinity active glucose transporters in the brush border. Because the affinity of the basolateral carrier is dependent on intracellular concentrations, carrier affinity does not necessarily have to increase in concert with the brush-border carrier. Also, it would be interesting to follow up on an earlier observation that the muscle/adipocyte isoform is present in the kidney (4). Because the liver is one of the few organs in which bidirectional glucose transport occurs across the same membrane, it would also be intriguing to see whether the sinusoidal membrane has more than one glucose transport protein.

A striking clinical correlate to the tissue distribution of glucose transport proteins is the distribution of the antineoplastic drug streptozotocin (STZ). STZ is a glucopyranoside that is taken up by liver, kidney, intestine and pancreatic $\beta$ cells, exactly corresponding to the distribution of liver-type glucose transporters. That the glucose moiety is important for the drug's activity is suggested by studies by Kawada et al. (11) who demonstrated that STZ analogs with altered sugar moieties were not taken up by tissues and were not cytotoxic. It is also known that the bactericidal effect of STZ is dependent on the presence of specific bacterial transport proteins, which take up the drug from the surrounding medium. STZ is cytotoxic for pancreatic $\beta$ cells in rats and proximal nephron in humans, both sites of the liver glucose transporter. The finding of liver-type glucose transport protein RNA in insulinoma cells is consistent with the therapeutic use of STZ in pancreatic islet-cell tumor. With antibodies and cDNA probes, it should be possible to confirm the hypothesis that STZ cytotoxicity is caused by the presence of a liver-type glucose transporter that facilitates entry of STZ into cells. 
In summary, the two papers show that the low-affinity liver isoform of glucose transporter is found in cells that primarily export glucose, such as those cells involved with transepithelial glucose transport or gluconeogenesis, whereas the high-affinity erythrocyte/brain transporter is found in cells that primarily take up glucose from plasma. Important and novel aspects of these studies include the demonstration of two types of transporters coexisting in the kidney, the type and density correlating with direction of transport (influx or efflux) and energy requirements, and the localization of a liver-type glucose carrier to the basolateral membrane of differentiated small intestinal surface cells, confirming their role in nutrient absorption. Unquestionably, a new and useful research tool for studying transmembrane glucose transport has been added to the investigator's armamentarium.

JONATHAN D. KAUNITZ, M.D.
Division of Gastroenterology
Department of Medicine
University of California at Los Angeles
Los Angeles, California

\section{REFERENCES}

1. Stevens BR, Kaunitz JD, Wright EM. Intestinal transport of amino acids and sugars: advances using membrane vesicles. Annu Rev Physiol 1984;46:417-433

2. Wheeler TJ, Hinkle PC. The glucose transporter of mammalian cells. Annu Rev Physiol 1985;47:503-517.

3. Hediger MA, Coady MJ, Ikeda TS, Wright EM. Expression cloning and cDNA sequencing of the $\mathrm{Na}$ '/glucose co-transporter. Nature 1987;330:379-380.

4. Birnbaum MJ. Identification of a novel gene encoding an insulinresponsive glucose transporter protein. Cell 1989;57:305-315.

5. Ciaraldi TP, Horuk R, Matthaei S. Biochemical and functional characterization of the rat liver glucose transport system. Biochem J 1986;240:115-123.

6. Cahill GF, Ashmore J, Earle AS, et al. Glucose penetration into liver. Am J Physiol 1958;192:491-496.

7. Birnbaum MJ, Haspel HC, Rosen OM. Cloning and characterization of a cDNA encoding the rat brain glucose-transporter protein. Proc Natl Acad Sci USA 1986;83:5784-5788.

8. Thorens B, Sarkar H, Kaback HR, Lodish HF. Cloning and functional expression in bacteria of a novel glucose transporter present in liver, intestine, kidney, and $\beta$-pancreatic islet cells. Cell $1988 ; 55: 281-290$.

9. Oka Y, Asano T, Sibasaki Y, et al. Increased liver glucosetransporter protein and mRNA in streptozocin-induced diabetic rats. Diabetes 1990;39:441-446.

10. Yamada Y, Seino Y, Takeda J, et al. Increase in liver glucose transporter mRNA levels during rat liver regeneration. Biochem Biophys Res Commun 1990;168:1274-1279.

11. Kawada J, Okita M, Nakatsuka M, Toyooka K, Naito S, Nabeshima A, Tsujihara K, et al. Ethylidene glucose-substituted new analogue of streptozotocin cannot induce diabetes: study on the basis of structure and activity relationship. Mol Cell Endocrinol 1989;62:153-159.

\section{TREATMENT OF HEPATOCELLULAR CARCINOMA}

Venook AP, Stagg Re, Lewis BJ, Chase JL, Ring EJ, Maroney TP, Hohn DC. Chemoembolization for hepatocellular carcinoma. J Clin Oncol 1990;8:1108-1114.

\section{ABSTRACT}

Fifty-one patients with unresectable hepatocellular carcinoma were treated by embolization of the hepatic artery with Gelfoam powder, contrast material and three chemotherapeutic agents (doxorubicin, mitomycin, cisplatin). Twelve patients (24\%) had a partial response with a decrease in the tumor diameter by at least $50 \%, 13$ patients $(26 \%)$ had only minor responses, $12(24 \%)$ had stabilization of disease and the remainder had progressive disease. Tumor liquefaction was noted on computed tomographic scanning in $70 \%$ of patients, and 23 of 34 patients with elevations in serum alphafetoprotein values had a greater than $50 \%$ reduction following treatment. The median patient survival time from treatment was 207 days. Most patients experienced transient pain, fever, nausea and elevations in serum aminotransferase activities as a result of therapy. Ascites developed in 14 patients. There were two treatment-related deaths: one from tumor hemorrhage and one from liver failure. Chemoembolization therefore appears to have significant activity in patients with hepatocellular carcinoma and is relatively well tolerated.

Franco D, Capussotti L, Smadja C, Bouzari H, Meakins $J$, Kemeny $F$, Grange $D$, et al. Resection of hepatocellular carcinoma: results in 72 European patients with cirrhosis. Gastroenterology 1990;98:733-738.

\section{ABSTRACT}

This study reports the results of resection in 72 cirrhotic patients with hepatocellular carcinoma from Europe. One and 3 year survival rates were $68 \%$ and $\mathbf{5 1 \%}$ respectively. Survival was significantly higher in Child's class $A$ than in class $B$ or $C$ patients. Patients with a thickly encapsulated tumor lived longer than those with an infiltrating tumor and also had a significantly lower recurrence rate. There was no relationship between the size of the tumor or the presence of symptoms and survival. These data suggest that good results can be achieved by resection of hepatocellular carcinoma in European cirrhotic patients. A thickly encapsulated tumor and good liver function are the main determinants of low cancer recurrence and high survival.

\section{COMMENTS}

HCC is one of the most common cancers worldwide, although it occurs relatively infrequently in the developed Western world (1). Typically HCC is seen at an advanced stage with extensive intrahepatic disease and metastases to lung, bone, adrenal gland or regional lymph nodes. It is also usually associated with significant liver disease, particularly cirrhosis (2). Because of this late presentation and association with cirrhosis, HCC has a grave prognosis, with survival times being measured in weeks to months. No effective therapy has been available for advanced HCC, although surgical resection has been considered potentially curative if carried out at an early stage. Within the past few years, a significant shift in emphasis has occurred with the attempt to diagnose HCC at an early, asymptomatic 